\title{
Questes
}

\section{La défiance dans La Belle Dame sans mercy d'Alain Chartier}

\section{Midoriko Kageyama}

\section{(2) OpenEdition}

\section{Journals}

\section{Édition électronique}

URL : http://journals.openedition.org/questes/1236

DOI : 10.4000/questes. 1236

ISSN : 2109-9472

\section{Éditeur}

Les Amis de Questes

\section{Édition imprimée}

Date de publication : 15 mars 2012

Pagination : 121-133

ISSN : 2102-7188

\section{Référence électronique}

Midoriko Kageyama, "La défiance dans La Belle Dame sans mercy d'Alain Chartier », Questes [En ligne], 23 | 2012, mis en ligne le 01 janvier 2014, consulté le 01 mai 2019. URL : http:// journals.openedition.org/questes/1236; DOI : 10.4000/questes.1236 


\title{
La défiance dans La Belle Dame sans mercy d'Alain Chartier
}

\author{
Midoriko KAGEYAMA
}

La Belle Dame sans mercy, rédigée par Alain Chartier dix ans après la défaite d'Azincourt ${ }^{1}$, fit scandale dans les milieux de la cour. Pour emprunter l'expression de Michel Zink, l'auteur dénonce «l'hypocrisie du jeu courtois $»^{2}$ dans ce poème lyrique ou débat en vers. Cette œuvre suscite des échos tels qu'elle produit plus tard tout un cycle de poèmes s'en inspirant. Le sujet est généralement considéré par la critique comme un défi aux valeurs de l'amour courtois. Ce poème emprunte une forme courante $\mathrm{au} \mathrm{XV}^{\mathrm{e}}$ siècle, le huitain à trois rimes enlacées, ababbcbc ${ }^{3}$. L'intrigue met en scène trois personnages : un amant plaintif qui déclare son amour, une dame impitoyable repoussant ses avances et un poète malheureux qui écoute leur conversation en cachette. Comme Daniel Poirion le fait remarquer, la combinaison de «l'amant-martyr» et de «la

\footnotetext{
${ }^{1}$ Pour la date de rédaction de ce poème, les opinions divergent. Cf. Arthur PIAGET, « La Belle Dame sans merci et ses imitations », Romania, 30 (1901), p. 22-48. Nous adoptons ici l'année 1424 retenue par A. Piaget.

${ }^{2}$ Michel ZINK, Introduction à la littérature française du Moyen Âge, Paris, LGF, « Le Livre de poche. Références », 500, 1993, p. 130.

3 Alain Chartier, La Belle Dame sans mercy et les poésies lyriques, Arthur PIAGET (éd.), Genève/Lille, E. Droz/Giard «Textes Littéraires Français », 1, 1949 (1 éd. 1945), p. viii (notre étude s'appuie sur cette deuxième édition, à laquelle le lexique de Robert-Léon Wagner est ajouté). Nous nous bornerons ici à relever deux œuvres prenant cette forme, le Testament de François Villon et le Champion des dames de Martin Le Franc. Un des grands rhétoriqueurs postérieurs à Alain Chartier, Jean Molinet, cite La Belle Dame sans merci comme un exemple typique de poème en huitains. Cf. Jean Molinet, «L'art de rhétorique », in Recueil d'arts de seconde rhétorique, Ernest LANGloIS (éd.), Paris, Imprimerie Nationale, "Collection de documents inédits sur l'histoire de France », 1902, p. 220 (réimpr. Genève, Slatkine Reprints, 1974).
} 
dame-sans-merci » n'est pas rare dans la littérature médiévale ${ }^{4}$. On retrouve également une situation analogue du poète dans le Débat de deux amans de Christine de Pizan $^{5}$. Ainsi, ce poème est formellement encadré par la tradition.

Pourtant, une opposition aussi constante de la Dame à l'Amant est remarquable parmi les textes de poésie lyrique où est mise en scène la «dame-sans-merci ${ }^{6}$. Arthur Piaget constate que, dans l'œuvre d'Alain Chartier, «tous les arguments de l'amoureux sont immédiatement réfutés ${ }^{7}$ par la Dame. Du début jusqu'à la fin, la Dame se défie des paroles de l'Amant, sans jamais changer d'attitude. Cette méfiance ferme et prolongée peut s'opposer aux sens étymologique et médiéval du doute : en

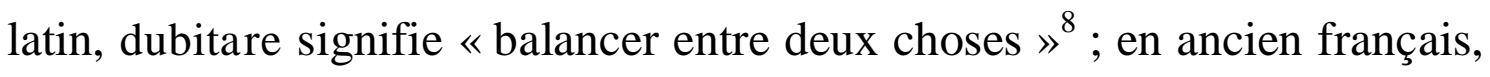
le doute renvoie à l'hésitation et (ou) à la crainte ${ }^{9}$. Cette spécificité de notre

${ }^{4}$ Daniel PoIRION, «Lecture de La Belle Dame sans mercy », in Mélanges de langue et de littérature médiévales offerts à Pierre Le Gentil, Paris, SEDES, 1973, p. 691-705.

${ }^{5}$ Dans le Débat de deux amans, un poète malheureux, étant arrivé au milieu d'une fête, écoute en cachette deux amants discutant d'amour. Cf. CHRISTINE DE PIZAN, Le Débat de deux amans, in Euvres poétiques de Christine de Pizan, Maurice RoY (éd.), Paris, Firmin Didot, 1891, « Société des anciens textes français », t. II, p. 49-109.

${ }^{6}$ Pierre Le Gentil constate que la «dame-sans-merci », en contraste avec sa beauté et ses qualités exceptionnelles, est «froide et insensible» dans la tradition depuis les troubadours. Mais il ajoute également que la dame ne refuse pas toujours le regard, ni même le baiser dans la lyrique française. Cf. Pierre LE Gentil, La Poésie lyrique espagnole et portugaise à la fin du Moyen Âge, t. I, Les Thèmes et les genres, Rennes, Plihon, 1949, p. 117.

7 Arthur Piaget, « La Belle Dame sans merci et ses imitations », art. cit., p. 25.

8 Cf. Dictionnaire illustré latin-français, Félix GAFFIOT (dir.), Paris, Hachette, 1934, entrée « dubito», p. 560.

9 Le «doute» prend au Moyen Âge le sens premier de «crainte, peur». Cf. Dictionnaire de l'ancienne langue française et de tous ses dialectes $d u I^{\mathrm{e}}$ au $X V^{\mathrm{e}}$ siècle, Frédéric GODEFROY (dir.), réimpr. Vaduz/Newyork, Scientific Periodicals Establishment/Kraus Reprint Corp., 1961, II, entrée «doute », p. 763. D’ailleurs, Pierre CROMER, dans le Dictionnaire du Moyen Français, fait la synthèse des significations de ce terme selon les deux axes suivants : l'«idée d'incertitude » et l'«idée de crainte ». Voir l'entrée «doute » dans l'édition électronique 2010 du Dictionnaire du Moyen 
texte est désignée par le terme que nous avons choisi, à savoir la défiance. La notion de défiance en moyen français (defiance, deffiance et desfiance) désigne à la fois le «défi » et la «défiance ». Le premier sens, « défi », implique l'«action de défier, de provoquer quelqu'un au combat, de déclarer la guerre à quelqu'un ». Le second sens est : « sentiment de celui qui n'a pas de confiance, manque de confiance, défiance $»^{10}$.

Notre étude porte également sur les motifs de défiance, faintise et change, qui figurent dans La Belle Dame sans mercy. En outre, à travers les verbes qui impliquent la confiance, croire et fier, nous traitons non seulement de la défiance explicite de la dame sans merci, mais aussi de celle de l'amoureux qui y correspond.

\section{Le commencement de la guerre amoureuse}

Pour exprimer l'innamoramento, les métaphores de la guerre sont souvent employées à la fin du Moyen Âge, comme en témoigne le refrain de la ballade CX de Charles d'Orléans : «Comme lassé de la guerre amoureuse $»^{11}$. Alain Chartier adopte des métaphores semblables dans une ballade où Desir et Doulz Regars, envoyés par Amours, attaquent le château du vouloir du poète ${ }^{12}$.

Dans La Belle Dame sans mercy, l'Amant, à travers le terme

Français, http://www.atilf.fr/dmf. Le Dictionnaire de l'ancienne langue française et le Dictionnaire du Moyen Français seront désormais abrégés respectivement en GDF et DMF.

10 Voir l'entrée «defiance » dans GDF, II, p. 460, et «desfiance» dans GDF, IX, p. 335. Voir aussi DMF.

11 Charles D’Orléans, Poésies, Pierre Champion (éd.), Paris, Champion, «CFMA », 34, 2010 ( $1^{\text {ère }}$ éd. 1923), t. 1, p. 171-172, v. 9, 18, 27 et 31.

12 Alain Chartier, La Belle Dame sans mercy et les poésies lyriques, éd. cit., Ballade IV, p. 51. 
deffiance, insiste sur le fait que les yeux de la Dame le provoquent à la guerre en lui envoyant un héraut représenté par Doubz Regart. Ici, la deffiance prend le sens de «défi » (au combat) ${ }^{13}$ en ancien français :

« Nully n'y pourroit la paix mettre

Fors vous qui la guerre y meistes

Quant vos yeulx escrivent la lettre

Par quoy deffier me feistes,

Et que Doubz Regart transmeistes,

Herault de celle deffiance,

Par lequel vous me promeistes

En deffiant bonne fiance. ${ }^{14}$

Le verbe deffier, associé au verbe causatif dans le vers 228, prend explicitement le sens de «provoquer ». D'autre part, dans le vers 232, il est possible de considérer que deffier comprend les deux sens de «provoquer » et de «se garder de » la bonne foi de l'Amant ${ }^{15}$. Autrement dit, le deuxième deffier connote le doute de la Dame qui fait l'objet de notre étude. La lettre écrite par les yeux et les messages transmis par Doubz Regart désignent la guerre verbale. Par contre, Desir qui figure dans la ballade d'Alain Chartier examinée plus haut n'est pas mis en scène ici.

Ainsi, il se peut que cette pièce soit interprétée comme une bataille d'idées entre deux combattants qui refusent toute confiance aux propos de l'adversaire ${ }^{16}$.

${ }^{13}$ Cf. GDF, II, entrée « defiance », p. 460.

14 Alain Chartier, La Belle Dame sans mercy et les poésies lyriques, éd. cit., p. 10, v. 225-232. Nous soulignons comme dans les citations suivantes.

15 Selon le Französisches Etymologisches Wörterbuch, le verbe defier signifie « retirer sa confiance à, se garder (de quelque chose, de quelqu'un)» en plus du sens de «provoquer (au combat)». Cf. Französisches Etymologisches Wörterbuch, Walther von WARTBURG (dir.), Tübingen, J.C.B. Mohr, 1949, III, entrée « fidare », p. 500a.

16 Daniel Poirion constate que deux thèses s'affrontent entre deux personnages enfermés dans leurs convictions. Cf. Daniel POIRION, «Lecture de La Belle Dame sans 


\section{Faintise et belles parolles}

Les termes caractérisant une langue nuisible, pour signifier la fausseté et la vanité de la parole, proviennent de la typologie des péchés de la langue. Celle-ci exerce une grande influence sur la réflexion morale à la fin du XII ${ }^{\mathrm{e}}$ siècle et au XIII ${ }^{\mathrm{e}}$ siècle ${ }^{17}$. Comme Danièle James-Raoul le fait remarquer, «l'ancien français développe un champ paradigmatique bien fourni exprimant ce mauvais usage de la langue $»^{18}$.

Héritiers de cette tradition, des termes de ce paradigme se retrouvent dans La Belle Dame sans mercy, tels que bourdes, belles paroles, mentir et mesdire $^{19}$, dont les deux premiers figurent dans le couplet suivant :

«Dames ne sont mie si lourdes,

Si malentendans ne si foles,

Que pour .j. poy de plaisans bourdes

Confites en belles parolles,

Dont vous autres tenez escolles

Pour leur faire croire merveilles,

Elles changent si tost leur colles.

A beau parler closes oreilles. ${ }^{20}$

Au début du débat, les «belles paroles » sont l'objet de la défiance de la Dame. Le choix de l'adjectif beau pour qualifier les paroles de l'Amant suggère la futilité des paroles des amoureux. La Dame généralise alors son discours en intégrant l'Amant dans la figure d'un interlocuteur collectif,

mercy », art. cit., p. 692.

17 Cf. Carla CASAgRande et Silvana Vecchio, Les Péchés de la langue, discipline et éthique de la parole dans la culture médiévale, Philippe BAILLET (trad.), Paris, Éditions du Cerf, « Histoire », 2007 ( $1^{\text {ère }}$ éd. italienne 1987), p. 17.

18 Danièle JAMES-RAOUL, La Parole empêchée dans la littérature arthurienne, Paris, Champion, « Nouvelle bibliothèque du Moyen Âge », 40, 1997, p. 66.

19 Alain Chartier, La Belle Dame sans mercy et les poésies lyriques, éd. cit., p. 13, v. 299,300 et 314 et p. 30 , v. 714.

${ }^{20}$ Ibid., p. 13, v. 297-304. 
dont elle se distancie avec l'expression dépréciative « vous autres ». Le proverbe au dernier vers de ce couplet conclut et renforce l'argument de la Dame.

Dans la suite du poème, l'Amant remplace le beau parleur auquel la Dame faisait allusion par le jangleur, celui qui se plaint par calcul :

«Il n'est jangleur, tant y meist

De sens, d'estudie ou de peine,

Qui si triste plainte feist

Comme cellui que le mal maine,

Car qui se plaint de teste saine

A peine sa faintise queuvre,

Mais pensee de douleur pleine

Preuve ses parolles par œuvre. $»^{21}$

L'Amant souligne le contraste qui existe entre un tel jangleur - qui ne sait guère dissimuler sa faintise (faux-semblant) - et un homme réellement triste. Aussi justifie-t-il l'authenticité de ses propres paroles. La Dame renchérit sur ce motif, employant l'expression «cruel losengeur». Par rapport à l'expression «plaisans bourdes » du couplet précédent, la notion de «mensonge » dément ici fortement la vérité des paroles : «Amours est cruel losengier, / Aspre en fait et doubz en mentir ${ }^{22}$. Les «belles parolles » sont également substituées aux «serments » qui désignent l'excès de la parole ainsi que sa fausseté :

«Ne cuidez que leurs sermens durent

Fors tant comme les mots se dient,

Et que Dieu et les sains s'en rient,

Car en telz sermens n'a riens ferme $[\ldots] »^{23}$.

21 Ibid., p. 13, v. 305-312.

22 Ibid., p. 13, v. 313-314.

${ }^{23}$ Ibid., p. 15, v. 347-350. Dana Symons fait remarquer qu'Alain Chartier dans ce passage insère le proverbe suivant: "Dieu et les saints se rient des serments des 
De plus, le thème qui lie la faintise à la parole est associé au cœur. La rhétorique oxymorique est utilisée parfois dans la poésie d'Alain Chartier ${ }^{24}$. Ici, l'antithèse s'applique au rapprochement du cœur et de la bouche. Au vers « loyal cuer et voirdisant bouche $»^{25}$ s'oppose le couplet suivant :

«Villain cuer et bouche courtoise

Ne sont mie bien d'une sorte.

Mais faintise tost les racoise

Qui par malice les assorte.

La mesnie Faulz Semblant s'aporte

Son honneur en sa langue fainte. $»^{26}$

La faintise atténue la divergence entre deux adjectifs, «villain» et « courtoise », à savoir qu'elle dissimule un cœur vil par des paroles courtoises. En outre, l'auteur lie la faintise à l'allégorie « Faulz Semblant», sous l'égide duquel même l'honneur, valeur chevaleresque, est véhiculé par la fausse langue.

La faintise de la parole est donc un fondement de la défiance de la Dame envers les paroles de l'Amant. En ce qui concerne les paroles, leur longueur est critiquée par la Dame, comme en témoigne la strophe consacrée aux «serments ». Par ailleurs, l'inconstance fait l'objet d'un débat entre la Dame et l'Amant.

amoureux ». Cf. Dana SYMONS, " "Car rompre vault pis que ployer": Proverbial Pleasure in La Belle Dame sans mercy », in Emma CAYLEY et Ashby KINCH (éd.), Chartier in Europe, Cambridge/Rochester, D. S. Brewer, « Gallica », 11, 2008, p. 45-59. ${ }^{24}$ Nous nous contentons ici de citer un exemple de ce style dans la chanson VI d'Alain Chartier : «Triste plaisir et doulereuse joie ». Cf. AlAin CHARTIER, La Belle Dame sans mercy et les poésies lyriques, éd. cit., p. 52, v. 1.

25 Ibid., p. 15, v. 358.

26 Ibid., p. 15, v. 361-366. 


\section{Change et « aller et venir »}

La Dame déprise la souffrance d'amour dont l'Amant se plaint, en l'attribuant à une «plaisant folie»:

«Legier cuer et plaisant folie,

Qui est meilleur quant plus est brieve,

Vous font ceste merencolie.

Mais c'est ung mal dont on relieve.

Faictes a voz pensees trieves $[\ldots] »^{27}$.

Cette «plaisant folie » était relevée par Charles d'Orléans, dans sa Ballade XCII où il faisait reconnaître aux jeunes gens leur arrogance ${ }^{28}$. L'état de cette folie, folie amoureuse, est ainsi désigné par Jacques Roubaud : «[...] il n'est que la conséquence extrême de l'“oubli de soi” que provoque l'amour $»^{29}$.

Contrairement à l'adresse précédente, par laquelle l'Amant était compris dans le collectif «vous autres $»^{30}$, la Dame lui adresse ici la parole en l'individualisant par l'emploi du mode impératif. Nous y retrouvons le terme militaire trieves. Afin de faire cesser les pensées amoureuses de l'Amant, la Dame propose littéralement à son adversaire une trêve.

En revanche, si la Dame adoucit son attitude, l'Amant la contredit en se comparant à des animaux de chasse apprivoisés :

«Qui a faucon, oysel ou chien

27 Ibid., p. 16, v. 377-381.

28 «Ce n'est fors que plaisant folie» (CHARLES D’OrlÉANS, Poésies, éd. cit., p. 146-147, refrain, v. 8, 16, 24 et 29).

29 Jacques RoubAUD, La Fleur inverse, l'art des troubadours, Paris, Les Belles Lettres, «Architecture du verbe », 2, 1994 (1 $1^{\text {ère }}$ éd. 1986), p. 68.

30 Alain Chartier, La Belle Dame sans mercy et les poésies lyriques, éd. cit., p. 13, v. 301 (cf. supra, «Faintise et belles parolles »). Notons que l'expression « vous et autres » se trouve au vers 345 . 
Qui le suit, aime, craint et doubte,

Il le tient chier et garde bien

Et ne le chace ne deboute.

Et je qui ay m'entente toute

En vous, sans faintise et sans change,

Suy rebouté plus bas qu'en soute

Et moins privé qu'ung tout estrange. » ${ }^{31}$

En se défendant de la double accusation de faintise et de change, l'Amant synthétise ici l'objet de la défiance de la Dame. La faintise a déjà été abordée plus haut, et le change renvoie au «changement en amour » et à «l'inconstance » en moyen français ${ }^{32}$. Le thème du change, en d'autres termes le fait de changer de maîtresse, s’oppose aux doctrines courtoises. Il ne paraît admis aux amoureux que dans le cas de la perte de leur maîtresse ${ }^{33}$.

L'amant se compare de nouveau avec un inconnu: «Je pers ce qu'estranges sy ont, / Donc me vaudroit ma loyauté / Moins qu'a ceulz qui viennent et vont $[. ..] »^{34}$. Le mouvement «aller et venir », exprimant la brièveté et l'instabilité, est adopté par la Dame pour décrire le cœur des amoureux :

«Leurs cuers s'en vont et puis reviennent,

31 Ibid., p. 16, v. 385-392.

32 Cf. DMF, entrée « change ».

33 Cf. Pierre LE GENTIL, La Poésie lyrique espagnole et portugaise à la fin du Moyen Âge, éd. cit., p. 159-160. Pierre Le Gentil analyse les concordances et les divergences sur les thèmes courtois entre la poésie française du Moyen Âge tardif et celles de l'Espagne et du Portugal. Le dieu d'Amour conseille parfois à un amant qui a perdu sa maîtresse d'en chercher une nouvelle, comme dans le cas de saint Valentin chez Oton de Grandson. Cf. OTON DE GRANDSON, «Complainte de Saint Valentin », in Oton de Grandson, sa vie et ses poésies, Arthur PIAGET (éd.), Lausanne, Payot, «Mémoires et documents publiés par la Société d'histoire de la Suisse romande », 1941, p. 187, v. 103-106.

34 Alain Chartier, La Belle Dame sans mercy et les poésies lyriques, éd. cit., p. 17, v. 402-404. 
Car ilz les ont bien reclamez

Et si bien apris qu'ilz retiennent

A changier desqu'ilz sont amez. » ${ }^{35}$

Le cœur est représenté dans ce contexte contradictoire par la métaphore, employée par l'Amant, des animaux de chasse tels que le faucon et le chien. Alors que l'Amant proteste en affirmant sa totale fidélité («sans change »), la Dame le réfute en employant la même métaphore et en substituant le verbe changer au substantif change.

C'est ainsi que la Dame développe dans ce poème son argument de la défiance en adoptant la méthode de son adversaire. Tandis qu'elle accuse l'Amant de faintise et de change, elle l'incite à croire ses propres paroles.

\section{Non croire}

Le refus de l'Amant de croire les propos de la Dame fait un parallélisme avec la défiance de la Dame. Une valeur de l'amour courtois, à savoir la «loyauté », fait l'objet de la foi de l'Amant ${ }^{36}$.

La Dame reproche à l'Amant de ne pas s'en rapporter à elle, comme en témoigne la phrase suivante : «Qui ne m'en croira, je m'en passe ${ }^{37}$. De son côté, l'Amant n'accepte pas le conseil de la Dame de trouver ailleurs une dame «plus belle et jente $»^{38}$, et n'ajoute pas non plus foi aux paroles de sa bien-aimée : «Le conseil que vous me donnez/Se puet mieulz dire qu'esploittier. / Du non croire me pardonnez ${ }^{39}$. Par contre,

\footnotetext{
35 Ibid., p. 25, v. 605-608.

${ }^{36} \mathrm{La}$ 《loyauté » renvoie à la «bonne foi ». Cf. GDF, V, p. 18.

37 Alain Chartier, La Belle Dame sans mercy et les poésies lyriques, éd. cit., p. 16, v. 384.

38 Ibid., p. 21, v. 490.

39 Ibid., p. 21, v. 497-499.
} 
l'amoureux a confiance en sa loyauté :

«Vous n'avez cause de doubter

Ne soupeczon qui vous esmeuve

A m'esloingner ne rebouter,

Car vostre bonté voit et treuve

Que j'ai fait l'essay et la preuve

Par quoy ma loyauté appert. » ${ }^{40}$

L'Amant prétend que la démonstration de sa loyauté peut dissiper le soupçon de la Dame. Cette démonstration procède par preuves et épreuves. Le thème de la «loyauté » est fréquent dans les œuvres d'Alain Chartier. La «loyauté », juxtaposée avec la «constance » au moment de s'adresser aux seigneurs $^{41}$, est soutenue jusqu'à la mort ${ }^{42}$.

En vain l'Amant essaie-t-il de convaincre la Dame, alors que l'accumulation par celle-ci des termes autour de la confiance est inefficace pour inciter celui-là à la croire :

«Et, se de vous n'avez pitié,

D'autri pitié ne vous fiez.

Mais soiez tout certiffiez

Que je sui celle que je fus. ${ }^{43}$

La guerre verbale entre l'amoureux et son « amoureuse annemie ${ }^{44}$ prend fin avec l'ultimatum de la Dame : «Une fois pour toutes croyez / Que vous

\footnotetext{
40 Ibid., p. 23, v. 545-549.

41 Cf. Alain ChartiER, Le Quadrilogue invectif, Eugénie DrOz (éd.), Paris, Librairie Ancienne Honoré Champion, « CFMA », 32, 1923, p. 14.

42 «Puis que ceulx pour loyaulx tenon / Qui se maintiennent / Si bien que foy et devoir tiennent / Vers leur seignieur et le [=le nom de Loyauté] soustiennent / Jusqu'au mourir, et entretiennent / Leur loyauté / Au besoing, et la fëauté / De leur dame et de sa beauté [...] » (Alain Chartier, «Le Livre des Quatre Dames », in The Poetical Works of Alain Chartier, James Cameron LAIDLAW (éd.), Cambridge/Londres, Cambridge University Press, 1974, p. 223, v. 848-855).

${ }^{43}$ Alain Chartier, La Belle Dame sans mercy et les poésies lyriques, éd. cit., p. 27 , v. 635-638.

44 Ibid., p. 24, v. 564.
} 
demourrez escondit. ${ }^{45}$. Nous pouvons interpréter le terme croire comme signifiant «être persuadé » ${ }^{46}$. Le verbe escondire signifie «refuser, repousser », en contexte amoureux. Ce terme remonte à l'étymon excondicere ${ }^{47}$, antonyme de condicere qui signifie «mettre en accord ».

En somme, ce débat se termine par un désaccord entre les deux interlocuteurs. En outre, le verbe demourer qui exprime la stabilité, souligne le caractère inébranlable des dispositions de la Dame.

Ainsi s'ouvre le débat entre la Dame et l'Amant par des métaphores militaires qui évoquent la guerre amoureuse, image d'un conflit intérieur. $\mathrm{Au}$ sujet du poème, Arthur Piaget fait remarquer la séparation des «préoccupations poétiques des courtisans » et des «événements extérieurs $»^{48}$. Pourtant, on ne saurait ignorer la cruauté de la Dame qui accule 1'Amant à la mort ${ }^{49}$. Cela pourrait être une allusion voilée aux conséquences désastreuses de la Guerre de Cent Ans ${ }^{50}$.

Ici se déroule une guerre verbale, sous forme de débat entre deux combattants qui ne se font pas confiance et refusent jusqu'à la fin de

\footnotetext{
45 Ibid., éd. cit., p. 32, v. 765-766.

46 Voir le lexique de Robert-Léon Wagner, ibid., p. 81.

${ }^{47}$ Cf. DMF, entrée « escondire ».

48 Arthur PiAGET, « La Belle Dame sans merci et ses imitations », art. cit., p. 23.

49 «Et depuis on me raporta / Qu'il avoit ses cheveulx deroups, / Et que tant se desconforta / Qu'il en estoit mort de courroux » (ALAIN CHARTIER, La Belle Dame sans mercy et les poésies lyriques, éd. cit., p. 33, v. 781-784).

50 Dans le Quadrilogue invectif, la nature impitoyable de l'armée d'Angleterre est soulignée à travers l'histoire. Cf. ALAIN CHARTIER, Le Quadrilogue invectif, éd. cit., p. 17-18 et p. 34-35. Alain Corbellari discute du reflet politique dans La Belle Dame sans mercy, en le mettant en rapport avec le Quadrilogue invectif. Il s'avère plutôt que la Dame est assimilée à la France. Cf. Alain CoRBELLARI, « À la place du mort. Une lecture politique de La Belle Dame sans Mercy», Le Moyen Français, 64 (2009), p. 55-66.
} 
reculer. Dans cette guerre verbale, bien différente de la bataille conforme au code chevaleresque, le fait de se rendre en demandant «merci » n'est pas accepté $^{51}$. Les requêtes formulées par l'Amant, aussi bien celles destinées à obtenir la «pitié » que la «grâce ${ }^{52}$, sont repoussées par la dureté de la Dame.

D'une part, la défiance de la dame sans merci porte entièrement sur la fausseté de la parole, faintise, énoncée par l'amoureux, ainsi que sur l'inconstance du cœur de ce dernier, le change. D'autre part, l'Amant met en doute, d'un bout à l'autre du poème, le refus de la Dame, en ne suivant pas son conseil. Même des vertus chevaleresques comme la loyauté, qui ne devraient pas être discutables, font l'objet du doute dans leur débat. À travers deux avis opposés, il semblerait que l'auteur vise à proposer à l'auditoire une mise en question des valeurs de la cour.

${ }^{51}$ Nous retrouvons fréquemment le chevalier ou sa suite qui demandent merci au vainqueur à la fin du combat chez Chrétien de Troyes. Cf. CHRÉTIEN DE Troyes, Les Romans de Chrétien de Troyes, t. 3, Le Chevalier de la Charrette, Mario RoQuES (éd.), Paris, Champion, "CFMA», 86, 1958, v. 2754-2849 et 898-931. Par ailleurs, conformément à la tradition de l'amant-martyr, l'Amant dans La Belle Dame sans mercy se plaint du manque de «merci » chez la Dame. Cf. Alain CHARTIER, La Belle Dame sans mercy et les poésies lyriques, éd. cit., p. 29, v. 690.

52 Ibid., p. 32, v. 753-758. 